\title{
Risk Factors for Total Tooth Loss in the United States; Longitudinal Analysis of National Data
}

\author{
Stephen A. Eklund, DDS, MHSA, DrPH \\ Brian A. Burt, BDS, MPH, PhD \\ Program in Dental Public Health \\ School of Public Health \\ University of Michigan \\ Ann Arbor, MI 48109-2029
}

\begin{abstract}
The NHANES / Epidemiologic Follow-up Study (NHEFS) of 1982-84 collected longitudinal data from 10,523 individuals initially seen during the first National Health and Nutrition Examination Survey (NHANES I) of 1971-75. Among this additional data was information on the incidence of total tooth loss during the 10 years between the surveys, which could then be added to NHANES I data to identify risk factors. In this analysis, a series of bivariate analyses were carried out, followed by logistic regression analysis to assess the simultaneous effect of major variables. Results showed that 7.4 percent of dentate Americans aged 25-74 at NHANES I became edentulous over the next 10 years. In bivariate analyses, the incidence of edentulism was correlated with baseline measures of lower income and education status, poorer oral health, self-perceptions of poor general health and oral health, absence of a regular dentist, and a lower number of remaining teeth at baseline. No correlation was found with gender and geographic region, nor with selfreported diabetes and arthritis, and age was not a factor when the number of remaining teeth at baseline were taken into account. In a logistic regression model assessing the effect of these variables simultaneously, none of the demographic variables retained significance; the only variable statistically significant in both age groups was the number of teeth remaining at baseline. Other significant variables in younger persons were higher periodontal disease scores, perceived poor dental health, perceived need for extractions, history of smoking, and low ascorbic acid intake. Some of these variables were reflections of negative health behavior and attitudes rather than direct correlates. Principal findings from this study were the importance of early tooth loss in eventual edentulism and the virtual disappearance of gender and age as determinants of total tooth loss.
\end{abstract}

Send correspondence and reprint requests to Dr. Eklund. This study was partially supported by a contract from the National Institute of Dental Research. Manuscript received: $12 / 9 / 93$; accepted for publication: $4 / 12 / 93$.
Key Words: edentulism, age, gender, tooth loss, attitudes, health behavior.

Well into the 20th century, total tooth loss among Americans was common (1-6). Improved tooth retention came with dental insurance that improved access to dental care, new technology such as the air-turbine handpiece, more positive attitudes toward tooth retention, and the benefits of modern preventive dentistry. By the time of the National Survey of Oral Health in US Employed Adults and Seniors in 1985-86, only 4.2 percent of employed adults under age 65 were edentulous, though the corresponding proportion for those aged 65 and older was 41.1 percent (7). It has been estimated that there will still be some 3 percent to 4 percent of Americans of all ages edentulous by the year 2024 (8).

Oral disease is not the only reason why people become edentulous; sociological and psychological factors also are involved (9). Data from several countries have consistently shown that edentulism is more prevalent among lower socioeconomic groups and among women, and that women have tended to become edentulous at a younger age (10-13). These gender differences are not easy to explain; many think they reflect dentist-patient relationships more than disease occurrence. They seem to be fading in the United States, however, for there was no apparent difference between employed men and women in 1985-86 (7). The strongest correlates with edentulism in that survey were age and socioeconomic status. Similar findings have been reported in regional studies in the United States (14).

While the picture of declining edentulism is encouraging, a better understanding of its underlying causes is still needed if the condition is to be minimized. Research on edentulism, however, is confined largely to cross-sectional analyses from which risk factors can only be inferred. An opportunity for longitudinal analysis, however, came with theNHANESI Epidemiologic Follow-up Study (NHEFS) of 1982-84, which collected additional data from some individuals initially seen during the first National Health and Nutrition Examination Survey 
(NHANES I) of 1971-75.* Dental data in NHEFS were restricted to issues of total tooth loss, which made it possible to identify those persons who became edentulous during the interval between the two surveys, approximately 10 years apart. Since extensive dental, medical, and social data were collected as part of NHANES I, we were able to seek the risk factors associated with total tooth loss during this 10 -year period. This report presents the results of those analyses.

\section{Data Sources: the NHANES I and NHEFS Surveys}

NHANES I 1971-75. The NHANES I survey was conducted by the National Center for Health Statistics (NCHS). It collected data from a probability sample of 20,749 individuals aged 1-74 years, representative of the civilian noninstitutionalized population of the coterminous United States except for those persons residing on American Indian lands $(15,16)$. Teams of interviewers and examiners traveled to each of 65 selected sample areas with mobile examination centers and conducted interviews and physical examinations under standardized conditions. Nutritional information came from a 24-hour dietary recall and a food frequency questionnaire; other health-related data came from general medical, dermatological, ophthalmological, dental, and anthropometric examinations, plus laboratory analyses of blood and urine samples.

A random subsample of 3,854 adults aged $25-74$ years within the overall NHANES I sample received a more detailed medical examination and provided more extensive questionnaire data on a variety of health conditions. The 1974-75 Augmentation Survey of 3,059 additional adults (also aged 25-74) further increased the number of adults for whom more extensive information was available (17). These two addenda to the main NHANES I thus provided detailed information from 6,913 adults aged 25-74 years.

Each participant in NHANES I (including those in the detailed substudy but not those in the Augmentation Survey) received a dental examination in which dental caries was recorded by the DMFT index (18), periodontal status by Russell's periodontal index (PI) (19), and plaque and calculus deposits by Greene and Vermillion's simplified oral hygiene index (OHI-S) (20). The dental examiners subjectively assessed each subject's treatment needs. Details on the dental examination in NHANES I have been reported elsewhere (21). In the 1974-75 Augmentation Survey, only a few questionnaire items on oral conditions and oral health care were included.

NHEFS 1982-84. NHEFS, also conducted by NCHS, was designed to investigate the association between baseline measures at NHANES I with the subsequent

*The main part of the NHANES I survey took place during the period 1971-74. An Augmentation Survey was also conducted during 1974-75. Because our analysis uses data from both of these parts of the survey, we use the time period 1971-75. development of specific diseases and conditions (22-24). The potential NHEFS study population was all 14,407 participants aged 25-74 years at baseline. Of these, 11,348 participants were from the 1971-74 NHANES I, and 3,059 from the 1974-75 Augmentation Survey.

The design of NHEFS consisted of (1) tracing the subjects or their proxies, (2) acquiring death certificates for those who had died, (3) performing in-depth interviews with subjects or their proxies, (4) taking physical measurements of subjects, and (5) obtaining the necessary hospital and nursing home records. Of the original target group of 14,407 adults, 13,380 (92.9\%) were successfully traced. There were $11,358(84.9 \%)$ of this group still living; interviews were conducted with $10,523(92.6 \%)$. The median follow-up period was 10 years. Data collected included interview information on health history since NHANES I, weight and blood pressure measurements, hospital and nursing home records, and death certificate information. Separate versions of the interview questionnaire were developed for subjects and proxies; proxy respondents completed 1,697 questionnaires for decedents.

Dentally related questions, repeated from NHANES I, were directed at the occurrence of the edentulous state, the use of dentures, and the use of fluoridated toothpaste. Only the questionnaire for live subjects contained the dental section. No dental examination was carried out as part of NHEFS.

\section{Methods}

The various combinations of data from examinations and questionnaire for those in NHANES I, the detailed substudy, and the Augmentation Survey mean that the number of participants included in different sections of this analysis will vary. Of the 10,523 interviewed in NHEFS, the 2,028 who were completely edentulous at NHANES I were excluded. This left 8,495 , of whom 6,480 were from NHANES I and 2,015 from the Augmentation Survey. Of the 6,480 dentate subjects from NHANES I (determined from the dental examination in that survey), data on dentition status were available for 6,405 at NHEFS. For the 2,015 subjects from the Augmentation Survey (who had no dental examination), baseline dentition status was ascertained from questionnaire responses, as was dentition status in NHEFS for all participants.

Most potential explanatory variables of interest to this analysis were available for the 6,405 dentate participants from NHANES I. Only the 3,854 adults in the detailed substudy and the 3,059 in the Augmentation Survey provided information on self-perception of dental, gingival, and general health; self-assessment of need for dental care; presence of a regular dentist and physician; reason for last dental visit; toothbrushing frequency; dental insurance; and smoking history. From these participants, there were 4,193 who were dentate at baseline, and for 
whom a full set of detailed baseline and follow-up interview data were available. In addition, 2,207 of this group from the detailed substudy of NHANES I also had baseline dental examination data available.

Subjects were considered to have become edentulous during the study period if they changed from dentate in one or both arches at NHANES I to edentulous in both arches at NHEFS. An edentulous arch was defined as one with no natural teeth or retained roots. Two questions in NHEFS sought information on the time since total tooth loss, but these questions were directed only to those few edentulous persons without a denture. Thus, the time over the 10-year period when people became edentulous was not available for most subjects.

With data sets as large as these, the analytical challenge is to maximize the value of the mass of information without being overwhelmed. Our approach was first to compute bivariate estimates, for each age group, of the incidence of total tooth loss over the 10-year period related to demographic factors, oral disease at baseline, selected variables in general health, health-related be-

TABLE 1

Weighted Estimates of the 10-year Incidence of Edentulism, Expressed as the Percent of 6,A05 Adults, by Sex and Age, US 1971-75 and 1982-84

\begin{tabular}{lccc}
\hline & \multicolumn{3}{c}{ Became Edentulous $(n=552)$} \\
\cline { 2 - 4 } Age & Male & Female & All \\
\cline { 3 - 4 } $25-34$ & 2.3 & 3.3 & 2.8 \\
$35-44$ & 5.0 & 6.7 & 5.9 \\
$45-54$ & 9.1 & 10.5 & 9.8 \\
$55-64$ & 13.7 & 9.6 & 11.6 \\
$65-74$ & 19.7 & 12.5 & 15.5 \\
All & 7.3 & 7.5 & 7.4 \\
& & & \\
\hline
\end{tabular}

TABLE 2

Weighted Estimates of the 10-year Incidence of Edentulism, Expressed as the Percent of 6,172 Adults, by Baseline Family Income and Age, US 1971-75 and 1982-84

\begin{tabular}{|c|c|c|c|c|}
\hline Age & $\begin{array}{c}<\$ 5,000 \\
(n=1,267)\end{array}$ & $\begin{array}{c}\$ 5,000- \\
\$ 9,999 \\
(n=2,093)\end{array}$ & $\begin{array}{c}\$ 10,000- \\
\$ 19,999 \\
(n=2,235)\end{array}$ & $\begin{array}{c}\$ 20,000+ \\
(n=577)\end{array}$ \\
\hline $25-34$ & 7.4 & 2.5 & 2.6 & 0.0 \\
\hline $35-44$ & 14.7 & 7.1 & 4.8 & 1.1 \\
\hline $45-54$ & 19.3 & 11.5 & 7.8 & 1.7 \\
\hline $55-64$ & 16.5 & 14.3 & 6.5 & $3.4^{*}$ \\
\hline $65-74$ & 18.4 & 15.7 & 12.2 & $8.2^{*}$ \\
\hline All & 15.1 & 8.2 & 5.2 & 1.7 \\
\hline
\end{tabular}

*Coefficients of variation of denominator $>0.15$. havior, and perceptions. These estimates were weighted, using sampling weights provided by NCHS, to adjust for disproportionate sampling in the original NHANES I design. We further adjusted these original weights to account for differential nonparticipation in the follow-up survey. We then used logistic regression analysis to assess the effect of these variables simultaneously. In the logistic regression analyses, the dentate/edentulous outcome was the dichotomous dependent variable. The coefficients are presented as log odds, so that those with a positive coefficient for the characteristic represented by the independent variable were more likely to become edentulous than those without it, while negative coefficients mean that individuals with the characteristic were less likely to become edentulous.

All of our statistical procedures used the sampling weights and the design structure of the NHANES I survey. A comprehensive evaluation of the design effects and analytic strategies specific to the NHANES I survey has been published (25). For Tables 1-8, the computer program \&PSALMS was used to estimate ratio means, and \&PSTABLE for subpopulation proportions. Both are available in the OSIRIS IV software package (26). Both

TABLE 3

Weighted Estimates of the 10-year Incidence of Edentulism, Expressed as the Percent of 6,365 Adults, by Individual Educational Level and Baseline Age, US 1971-75 and 1982-84

\begin{tabular}{lcccc}
\hline Age & $\begin{array}{c}<12 \text { Years } \\
(n=2,288)\end{array}$ & $\begin{array}{c}12 \text { Years } \\
(n=2,418)\end{array}$ & $\begin{array}{c}13 \text { Years+ } \\
(n=1,659)\end{array}$ \\
\cline { 1 - 2 } $25-34$ & 5.8 & & 3.2 & 1.0 \\
$35-44$ & 14.8 & & 3.6 & 1.6 \\
$45-54$ & 14.7 & & 9.7 & 3.8 \\
$55-64$ & 18.6 & & 9.0 & 2.0 \\
$65-74$ & 16.7 & & 16.1 & 12.8 \\
All & 14.2 & & 6.0 & 2.5
\end{tabular}

TABLE 4

Weighted Estimates of the 10-year Incidence of Edentulism, Expressed as the Percent of 6,405 Adults, by Geographic Region and Baseline Age, US 1971-75 and 1982-84

\begin{tabular}{|c|c|c|c|c|}
\hline Age & $\begin{array}{l}\text { Northeast } \\
(n=1,393)\end{array}$ & $\begin{array}{l}\text { Midwest } \\
(n=1,617)\end{array}$ & $\begin{array}{c}\text { South } \\
(n=1,661)\end{array}$ & $\begin{array}{c}\text { West } \\
(n=1,734)\end{array}$ \\
\hline $25-34$ & 2.6 & 3.7 & 3.8 & 0.9 \\
\hline $35-44$ & 4.3 & 4.8 & 11.7 & 3.5 \\
\hline $45-54$ & 9.2 & 10.5 & 12.1 & 7.5 \\
\hline $55-64$ & 14.8 & 11.4 & $13.6^{*}$ & 5.7 \\
\hline $65-74$ & 20.0 & 13.3 & 17.4 & 11.6 \\
\hline All & 7.9 & 7.2 & 10.1 & 4.6 \\
\hline
\end{tabular}

*Coefficients of variation of denominator $>0.15$. 
TABLE 5

Weighted Estimates of 10-year Incidence of Edentulism, Expressed as Percent of 6,405 Adults, by Baseline Age and Number of Remaining Teeth at Baseline, US 1971-75 and 1982-84

\begin{tabular}{lcccc}
\hline & \multicolumn{4}{c}{ Number of Teeth } \\
\cline { 2 - 5 } Age & $\begin{array}{c}1-7 \\
(n=275)\end{array}$ & $\begin{array}{c}8-15 \\
(n=869)\end{array}$ & $\begin{array}{c}16-23 \\
(n=1,258)\end{array}$ & $\begin{array}{c}24+ \\
(n=4,003)\end{array}$ \\
\cline { 5 - 5 } $25-34$ & $48.9^{*}$ & 15.8 & 5.2 & 1.4 \\
$35-44$ & $28.9^{*}$ & 18.8 & 7.5 & 2.1 \\
$45-54$ & $50.9^{*}$ & 29.5 & 8.2 & 2.8 \\
$55-64$ & $30.6^{*}$ & 27.2 & 12.4 & 3.3 \\
$65-74$ & 59.6 & 19.4 & 11.9 & 2.3 \\
All & 41.8 & 23.2 & 8.8 & 2.1 \\
\hline
\end{tabular}

TABLE 6

Weighted Estimates of 10-year Incidence of Edentulism, Expressed as Percent of 6,401 Adults, by Baseline Age and Periodontal Status, US 1971-75 and 1982-84

\begin{tabular}{lcccc}
\hline Age & $\begin{array}{c}\text { Healthy } \\
(n=3,135)\end{array}$ & $\begin{array}{c}\text { Gingivitis } \\
\text { Only } \\
(n=1,455)\end{array}$ & $\begin{array}{c}1-3 \\
\text { Pockets* } \\
(n=473)\end{array}$ & $\begin{array}{c}\text { 4 or More } \\
\text { Pockets* } \\
(n=1,338)\end{array}$ \\
\hline $25-34$ & 1.1 & 2.7 & $3.8+$ & 12.7 \\
$35-44$ & 2.5 & 3.9 & 11.3 & 16.6 \\
$45-54$ & 6.6 & 7.9 & $14.5+$ & 15.4 \\
$55-64$ & 3.9 & $8.1+$ & $20.5+$ & 22.0 \\
$65-74$ & 9.4 & 11.9 & 26.1 & 20.5
\end{tabular}

"More than $3 \mathrm{~mm}$.

tCoefficients of variation of denominator $>0.15$.

${ }^{*}$ Coefficients of variation of denominator $>0.15$

TABLE 7

Weighted Estimates of Mean DMFT Scores and Individual Components of Decayed, Missing, and Filled Teeth for 6,405 Adults, by Change in Dentition Status and Baseline Age, US 1971-75 and 1982-84

\begin{tabular}{|c|c|c|c|c|c|c|c|c|}
\hline \multirow[b]{2}{*}{ Age } & \multicolumn{4}{|c|}{ Remained Dentate $(n=5477)$} & \multicolumn{4}{|c|}{ Became Edentulous $(n=552)$} \\
\hline & DMFT & $\mathrm{D}$ & $\mathbf{M}$ & $\mathrm{F}$ & DMFT & $\mathrm{D}$ & $\mathbf{M}$ & $\mathbf{F}$ \\
\hline $25-34$ & 15.0 & 1.5 & 3.9 & 9.5 & $22.4^{*}$ & $4.2^{*}$ & $16.1^{*}$ & $2.1^{*}$ \\
\hline $35-44$ & 19.3 & 1.0 & 8.2 & 10.0 & 22.6 & 2.1 & 18.3 & 2.2 \\
\hline $45-54$ & 19.2 & 0.8 & 9.2 & 9.2 & 24.4 & 1.2 & 20.1 & 3.1 \\
\hline $55-64$ & 20.5 & 0.9 & 10.9 & 8.7 & 23.8 & 1.4 & 19.3 & 3.1 \\
\hline $65-74$ & 21.1 & 0.6 & 12.7 & 7.9 & 26.1 & 0.7 & 22.8 & 2.6 \\
\hline
\end{tabular}

${ }^{*}$ Coefficients of variation of denominator $>0.15$.

routines utilize a linearized Taylor Series expansion, which can be unstable when the sampling error computing unit is small. Instances where interpretive caution is warranted because estimators have a coefficient of variation for the denominator of greater than 0.15 have been noted in the tables.

The logistic models in Tables 9, 10, and 12 were all produced using the PC CARP software (27), which is intended for multistage stratified samples like those employed in NHANES I. The design effect values in these tables are the ratio of the variance for a statistic from the complex sample to the corresponding variance from a simple random sample design. The SAS PROC LOGIST command (28) was used to calculate the $R^{2}$ values in these tables, which are the proportions of log likelihood explained by each model.

\section{Results}

Bivariate Relationships. Table 1 shows that 7.4 percent of dentate adult Americans became edentulous in the 10 years between the two surveys. Tables $2-4$ show the incidence of edentulism by family income, individual education, and geographic region, respectively. Income-
TABLE 8

Weighted Estimates of 10-year Incidence of Edentulism, Expressed as Percent of 4,157 Adults, by Baseline Age and Perceived Dental Health Status, US 1971-75 and 1982-84

\begin{tabular}{|c|c|c|c|c|}
\hline Age & $\begin{array}{c}\text { Excellent } \\
(n=520)\end{array}$ & $\begin{array}{c}\text { Good } \\
(n=1,756)\end{array}$ & $\begin{array}{c}\text { Fair } \\
(n=1,207)\end{array}$ & $\begin{array}{c}\text { Poor } \\
(n=674)\end{array}$ \\
\hline $25-34$ & 0.4 & 1.3 & 1.8 & 11.1 \\
\hline $35-44$ & 0.7 & 2.0 & 6.9 & 25.3 \\
\hline $45-54$ & 2.3 & 3.2 & 7.8 & 32.8 \\
\hline $55-64$ & 1.1 & 5.6 & 7.5 & 33.4 \\
\hline $65-74$ & $1.6^{*}$ & 5.7 & 7.5 & 33.4 \\
\hline All & 1.0 & 2.8 & 5.9 & 25.4 \\
\hline
\end{tabular}

${ }^{*}$ Coefficients of variation of denominator $>0.15$.

related differences were even more pronounced than the data in Table 2 suggest, because more of the lower income groups were already edentulous at baseline. Similar patterns are seen in Table 3, relating incidence of edentulism to education. There were no clear patterns by geographic region (Table 4). 
TABLE 9

Effect of Number of Remaining Teeth at Baseline on the Incidence of Total Tooth Loss Among 6,A05 Adults, by Age at Baseline, US 1971-74 and 1982-84

\begin{tabular}{|c|c|c|c|c|}
\hline Variable & Regression Coefficient & SE & $T$-statistic & Design Effect \\
\hline \multicolumn{5}{|l|}{ Age $25-59(n=5,132) ; R^{2}=0.231$} \\
\hline \multicolumn{5}{|l|}{ Independent variable } \\
\hline Number of remaining teeth & -0.137 & 0.012 & $11.78+$ & 2.236 \\
\hline \multicolumn{5}{|l|}{ Covariables } \\
\hline Age & 0.024 & 0.009 & $2.74+$ & 1.671 \\
\hline Nonwhite indicator & -0.426 & 0.208 & $2.05^{*}$ & 1.126 \\
\hline Female indicator & -0.047 & 0.167 & 0.28 & 1.803 \\
\hline Income $<\$ 5,000$ & 0.721 & 0.194 & $3.71+$ & 1.686 \\
\hline Education $<12$ years & 0.835 & 0.222 & $3.76+$ & 2.969 \\
\hline South indicator & 0.337 & 0.225 & 1.50 & 2.750 \\
\hline \multicolumn{5}{|l|}{ Age $60-74(n=1,273) ; R^{2}=0.265$} \\
\hline \multicolumn{5}{|l|}{ Independent variable } \\
\hline Number of remaining teeth & -0.185 & 0.021 & $8.98+$ & 1.853 \\
\hline \multicolumn{5}{|l|}{ Covariables } \\
\hline Age & 0.047 & 0.030 & 1.53 & 1.426 \\
\hline Nonwhite indicator & 0.983 & 0.307 & $3.20+$ & 1.065 \\
\hline Female indicator & -0.743 & 0.315 & $2.36^{*}$ & 2.373 \\
\hline Income $<\$ 5,000$ & 0.020 & 0.224 & 0.09 & 0.995 \\
\hline Education $<12$ years & -0.052 & 0.268 & 0.19 & 1.548 \\
\hline South indicator & 0.050 & 0.337 & 0.15 & 2.117 \\
\hline
\end{tabular}

*Denotes statistical significance at the $5 \%$ level. tDenotes statistical signficance at the $1 \%$ level.

Among the baseline dental conditions, Table 5 shows that the number of teeth remaining at baseline was strongly associated with the incidence of edentulism. Nearly 42 percent of those with only $1-7$ teeth remaining at baseline were edentulous 10 years later, compared to just over 2 percent of those with 24 or more teeth at baseline. Table 5 also shows that the 10-year incidence of edentulism is remarkably stable by age within each group. Young adults with few remaining teeth were just as likely to become edentulous as older adults with the same number of remaining teeth. In addition, older adults with most of their teeth at baseline were no more likely to become edentulous than younger adults with the same number of teeth at baseline.

Other bivariate relationships between the incidence of edentulism and oral health variables revealed no unexpected findings. Those with poorer periodontal health, as measured by the number of pockets of 3 mm or more (i.e., PI scores of 6 or 8 ), had a relatively greater incidence of edentulism (Table 6). So did those with a greater DMFT score at baseline (Table 7), where the D components did not show much difference across the groups, but the $M$ and $\mathrm{F}$ components showed big relative differences. Table 8 shows a high level of concordance between self-perceptions of oral health and the incidence of edentulism; those who considered their dental health to be excellent were unlikely to become edentulous at any age. Other findings, for which data were not tabulated, included a greater incidence of edentulism in persons (1) with more extensive deposits of plaque and calculus, (2) who reported a lower frequency of toothbrushing, (3) whose most recent dental visit was more than one year ago, (4) with more extensive professionally defined need for treatment, (5) who perceived their general health to be poorer, and (6) who reported having no regular dentist (though interestingly enough this pattern was not seen with those reporting no regular physician). The relationship with the level of reported alcohol consumption was erratic, and that with dental insurance was not pursued since dental insurance was uncommon at the time of NHANES I.

Some relationships with general health conditions were explored, but revealed little of interest. There were too few known diabetics in the sample to permit conclusions. Among the nearly 20 percent of the sample who reported having arthritis, there was a slightly greater incidence of edentulism; however, the differences did not attain statistical significance. A general pattern of higher levels of nutrient intake was seen among those who remained dentate, but none reached statistical signifi- 
TABLE 10

Effect of Selected Oral Health Conditions at Baseline on the Incidence of Total Tooth Loss Among 6,A05 Adults, by Age at Baseline, US 1971-74 and 1982-84

\begin{tabular}{|c|c|c|c|c|}
\hline Variable & Regression Coefficient & $\mathrm{SE}$ & $T$-statistic & Design Effect \\
\hline \multicolumn{5}{|l|}{ Age $25-59(n=5,132) ; R^{2}=0.317$} \\
\hline \multicolumn{5}{|l|}{ Independent variable } \\
\hline Number of remaining teeth & -0.126 & 0.021 & $5.94^{\mathbb{I}}$ & 2.632 \\
\hline Periodontal index & 0.160 & 0.041 & $3.91^{\text {II }}$ & 1.459 \\
\hline Oral hygiene index & 0.174 & 0.065 & $2.69^{\text {II }}$ & 1.504 \\
\hline DMF & 0.008 & 0.021 & 0.38 & 1.592 \\
\hline$\#$ caries $^{*}$ & 0.123 & 0.032 & $3.79^{\mathbb{I}}$ & 1.795 \\
\hline \#periot & 0.089 & 0.026 & $3.37^{\text {II }}$ & 1.821 \\
\hline \# other $¥$ & 0.124 & 0.040 & $3.13^{\mathrm{gl}}$ & 1.087 \\
\hline \multicolumn{5}{|l|}{ Covariables } \\
\hline Age & 0.025 & 0.009 & $2.68^{\text {I }}$ & 1.637 \\
\hline Nonwhite indicator & -0.636 & 0.190 & $3.35^{\mathbb{I I}}$ & 0.797 \\
\hline Female indicator & 0.242 & 0.178 & 1.36 & 1.725 \\
\hline Income $<\$ 5,000$ & 0.446 & 0.193 & $2.32^{\S}$ & 1.411 \\
\hline Education $<12$ years & 0.527 & 0.242 & $2.18^{\S}$ & 2.911 \\
\hline South indicator & 0.247 & 0.228 & 1.08 & 2.459 \\
\hline \multicolumn{5}{|l|}{ Age $\left.60-74(n=1,273) ; R^{2}=0.319\right)$} \\
\hline \multicolumn{5}{|l|}{ Independent variable } \\
\hline Number of remaining teeth & -0.175 & 0.029 & $5.94^{\text {I }}$ & 1.431 \\
\hline Periodontal index & 0.180 & 0.060 & $3.01^{\text {II }}$ & 1.797 \\
\hline Oral hygiene index & 0.177 & 0.099 & 1.78 & 1.392 \\
\hline DMF & 0.009 & 0.048 & 0.19 & 1.869 \\
\hline \# caries* & -0.012 & 0.058 & 0.21 & 1.142 \\
\hline \# periot & 0.048 & 0.051 & 0.95 & 1.852 \\
\hline \# otherf & 0.157 & 0.046 & $3.44^{\text {II }}$ & 0.808 \\
\hline \multicolumn{5}{|l|}{ Covariables } \\
\hline Age & 0.063 & 0.033 & 1.90 & 1.456 \\
\hline Nonwhite indicator & 0.405 & 0.243 & 1.67 & 0.543 \\
\hline Female indicator & -0.631 & 0.337 & 1.87 & 2.300 \\
\hline Income $<\$ 5,000$ & 0.064 & 0.228 & 0.28 & 0.915 \\
\hline Education $<12$ years & -0.176 & 0.283 & 0.62 & 1.496 \\
\hline South indicator & -0.006 & 0.381 & 0.01 & 2.390 \\
\hline
\end{tabular}

Number of extractions indicated due to caries.

† Number of extractions indicated due to periodontal disease.

N Number of extractions indicated due to other reasons.

${ }^{5}$ Denotes statistical significance at the $5 \%$ level.

IDenotes statistical significance at the $1 \%$ level.

cance.

Multivariate Relationships. Logistic regression analyses were first carried out to test the simultaneous effect of the demographic variables assessed individually in Tables 1-4, with the addition of a race/ethnic indicator. The dependent variable was coded 0 for those who remained dentate after 10 years, and 1 for those who became edentulous in both arches. Analyses were carried out separately for those aged 25-59 and 60-74 to allow for the possibility that total tooth loss in older individuals might be a fundamentally different process from that in younger persons. The results of this first multivariate analysis showed that in the 25-59 age group, age, lower income and education status, and residence in the Midwest and South remained associated with the likelihood of becoming edentulous. Contrasting patterns, however, were found in those aged 60-74.

Table 9 assesses the effect of the number of remaining teeth on the incidence of total tooth loss, while controlling for six potential confounders identified in the initial multivariate analysis. The purpose of this analysis was to get a better assessment of the role of the independent vari- 
TABLE 11

Independent Variables that Retained Significance and Those That Did Not, after Inclusion in Logistic Regression Model Shown in Tables 9 and 10

Retained Significance
Periodontal index scores
Number of remaining teeth
General need for dental treatment
Perception of poor dental health
Perceived need for extraction(s)
Having a regular dentist
Denture as reason for last dental visit
Last cleaning more than 1 year ago
Ever smoked
Dietary protein
Dietary ascorbic acid

Lost Significance

Perceived poor gingival health

Perceived need for treatment other than extractions

Perceived poor general health

Self-reported arthritis

Reason for last dental visit being other than denture preparation

Having a regular physician

Last dental visit more than 1 year ago

Ever having had a professional cleaning

All levels of alcohol consumption

Dietary variables other than protein and ascorbic acid

able (remaining teeth), and not necessarily to explore the independent effects of the covariables. Table 9 shows that the number of remaining teeth at baseline remained strongly associated with the incidence of edentulism, even when the potential confounders were included. The negative sign of the regression coefficient means that the more teeth present, the less likely the individual was to become edentulous during the 10-year interval. The role of remaining teeth is quite similar in both the younger and the older group, and the level of statistical significance remains high in both.

A series of regression models (not tabulated) with the same set of six covariables shown in Table 9 was used to test the role of other independent variables examined in the bivariate analyses. Among the oral health variables, statistically significant relationships $(P<.01)$ with the incidence of total tooth loss were retained in the younger age group by PI scores, mean DMFT, the D component and $\mathrm{M}$ component of DMFT, and the number of teeth indicated for extraction. OHI-S scores were also significant $(P<.05)$. In the older age group the results were similar, except the OHI-S scores were not significant and the $\mathrm{D}$ component was significant at $P<.05$.

Table 10 shows the regression model with the oral health variables that remained statistically significant, included as independent variables. The coefficient for the number of remaining teeth remained virtually unchanged despite the inclusion of other oral health variables, and its statistical significance remained high in both age groups. The coefficient for the OHI-S score also remained stable, though it was statistically significant only in the younger age group. The coefficient for the PI was greatly diminished, although in both age groups it maintained statistical significance. The coefficient for the DMF index dropped to practically zero, indicating that with the other independent variables in the model the information in the DMF variable contributed little to the model's ability to predict the incidence of edentulism.

We continued this analytic approach, using the same logistic regression model, with the same set of six covariables, to evaluate as independent variables all the other factors examined in the bivariate analyses. Table 11 lists the variables that continued to appear as important predictors in these analyses, and those that did not. The important predictor variables were then included as independent variables in an additional logistic regression model. Since this combination of variables was collected only for subjects in NHANES I and the detailed substudy, the group size in this analysis was reduced to 2,207. Results are shown in Table 12, where it can be seen that none of the demographic covariables attained statistical significance in either age group. The only variable statistically significant in both age groups was the number of teeth remaining at baseline. Other significant variables in the younger age group were PI score, perceived poor dental health, perceived need for extractions, history of smoking, and low ascorbic acid intake.

\section{Discussion}

Analysis of large data sets can provide valuable insights that could not be obtained any other way, though the limitations of this kind of study are recognized. One is that participants from NHANES I who were included in NHEFS were generally younger and better educated than the original sample, a form of selection bias that is inherent in all follow-up studies. Second, analytic power is reduced because (1) those who became edentulous early in the 10-year period cannot be distinguished from those who became edentulous later; and (2) a person who had many extractions during the interval, stopping just short of edentulism, would be classified the same way as one who lost no teeth at all. A theoretically complete model of tooth loss would include an assessment of the patterns and timing of loss of individual teeth. A third 
TABLE 12

Effect of Selected Risk Factors on the Incidence of Total Tooth Loss among 2,207 Adults, by Age at Baseline, US 1971-75 and 1982-84: Model with 12 Independent Variables and 6 Covariables

\begin{tabular}{|c|c|c|c|c|}
\hline Variable & Regression Coefficient & SE & $T$-statistic & Design Effect \\
\hline \multicolumn{5}{|l|}{ Age $\left.25-59(n=1,819) ; \mathrm{R}^{2}=0.308\right)$} \\
\hline \multicolumn{5}{|l|}{ Independent variable } \\
\hline Number of remaining teeth & -0.100 & 0.031 & $3.28 \ddagger$ & 3.837 \\
\hline Periodontal index & 0.293 & 0.066 & $4.45 \ddagger$ & 1.684 \\
\hline General treatment need & -0.319 & 0.384 & $0.83^{\circ}$ & 1.155 \\
\hline Poor dental health & 0.650 & 0.264 & $2.46 \ddagger$ & 0.845 \\
\hline Perceived need for extraction & 0.870 & 0.308 & $2.83 \ddagger$ & 1.514 \\
\hline Having regular dentist & -0.142 & 0.285 & 0.50 & 1.345 \\
\hline Denture as reason for last visit & 0.208 & 0.457 & 0.45 & 2.520 \\
\hline Last cleaning $\geq 1$ year & 0.128 & 0.306 & 0.42 & 1.462 \\
\hline Ever smoke & 0.630 & 0.300 & $2.10+$ & 1.412 \\
\hline Protein ${ }^{*}$ & -0.0017 & 0.0026 & 0.65 & 1.184 \\
\hline Ascorbic acid* & -0.0031 & 0.0011 & $2.71 \ddagger$ & 1.064 \\
\hline \multicolumn{5}{|l|}{ Covariables } \\
\hline Age & 0.028 & 0.016 & 1.74 & 1.759 \\
\hline Nonwhite indicator & -0.462 & 0.263 & 1.76 & 0.632 \\
\hline Female indicator & 0.467 & 0.311 & 1.50 & 1.621 \\
\hline Income $<\$ 5,000$ & 0.465 & 0.357 & 1.30 & 1.812 \\
\hline Education $<12$ years & 0.008 & 0.350 & 0.02 & 2.161 \\
\hline South indicator & 0.334 & 0.336 & 0.99 & 2.039 \\
\hline \multicolumn{5}{|l|}{ Age $\left.60-74(n=388) ; R^{2}=0.193\right)$} \\
\hline \multicolumn{5}{|l|}{ Independent variable } \\
\hline Number of remaining teeth & -0.173 & 0.048 & $3.61+$ & 2.235 \\
\hline Periodontal index & 0.091 & 0.101 & 0.90 & 1.524 \\
\hline General treatment need & 0.725 & 0.700 & 1.04 & 1.264 \\
\hline Poor dental health & 0.074 & 0.658 & 0.11 & 1.967 \\
\hline Perceived need for extraction & 1.070 & 0.658 & 1.72 & 1.566 \\
\hline Having regular dentist & -0.191 & 0.616 & 0.31 & 1.675 \\
\hline Denture as reason for last visit & -0.066 & 0.579 & 0.11 & 1.607 \\
\hline Last cleaning $\geq 1$ year & -0.640 & 0.669 & 0.96 & 2.226 \\
\hline Ever smoke & -0.355 & 0.553 & 0.64 & 1.684 \\
\hline Protein ${ }^{*}$ & 0.0012 & 0.0061 & 0.20 & 1.888 \\
\hline Ascorbic acid* & 0.0006 & 0.0013 & 0.44 & 1.563 \\
\hline \multicolumn{5}{|l|}{ Covariables } \\
\hline Age & 0.088 & 0.051 & 1.71 & 1.029 \\
\hline Nonwhite indicator & 0.340 & 0.712 & 0.48 & 1.403 \\
\hline Female indicator & -0.767 & 0.521 & 1.47 & 1.445 \\
\hline Income $<\$ 5,000$ & -0.106 & 0.683 & 0.16 & 2.299 \\
\hline Education $<12$ years & -0.374 & 0.680 & 0.55 & 2.231 \\
\hline South indicator & 0.354 & 0.720 & 0.49 & 2.594 \\
\hline
\end{tabular}

"Using percent of NHANES standards for each dietary component.

tDenotes statistical significance at the $5 \%$ level.

tDenotes statistical significance at the $1 \%$ level.

limitation is having to use disease measures from NHANES I that are not used today. The PI, for example, is no longer considered a valid measure of periodontal status (9), and the OHI-S index has also rarely been used in recent years.

Even with these limitations, the study yields valuable information from a large group that approximates a nationally representative sample. In the bivariate assess- 
ments (Tables 1-8), many of the associations previously seen in cross-sectional data were confirmed. The similarity between men and women in the low rates of total tooth loss in the three younger age groups (Table 1 ), seen also in the prevalence data from the 1985-86 adult survey (7), show that the historic difference in edentulism between the sexes has largely disappeared. It also reflects the overall decline in tooth loss among younger people. Lower income and education, however, were still highly associated with total tooth loss, regardless of age (Tables 2-3). Despite the overall decline in edentulism, the poor and less-educated of all ages continued to be much more likely to become edentulous. Positive bivariate associations between edentulism and PI scores (Table 6), OHI-S scores, and DMFT values (Table 7) were expected, as they were with self-perceived oral health status (Table 8) and professionally defined treatment needs. Smoking and ascorbic acid intake both showed some bivariate associations with edentulism, though their apparent importance declined in the multivariate models.

Among the baseline oral conditions related to total tooth loss, the most convincing association came with the number of remaining teeth (Table 5). Those with 1-7 teeth at baseline were nearly 20 times more likely to become totally edentulous than those with 24 or more teeth, and this relative risk was unaffected by age. This means that the youngest people with few teeth were just as likely to become edentulous as the oldest people with few teeth. Conversely, the oldest people who had most of their teeth at baseline were no more likely to become edentulous than the youngest people with the same number of teeth. In Table 5, the association between age and the incidence of edentulism virtually disappears when controlling for the number of teeth present at baseline. In the multivariate analyses (Tables 9-12), the number of remaining teeth at baseline stands out as a remarkably stable predictor of edentulism, even when many other variables were assessed simultaneously. In these multivariate analyses, no demographic variable (i.e., age, race, sex, place of residence, income, education) emerged as a significant predictor of edentulism when the number of remaining teeth at baseline was included in the model.

The relatively short time period over which incidence was measured (10 years) enhances the dominance of the variable for the number of remaining teeth at baseline. This variable has tied up within it the cumulative effect of all the disease experience and treatment choices made through the years; tooth loss predicts tooth loss. Sorting out the roles of the likely causes of the prior extractions would require study of a different type of data set, one that follows the dentitions of young people through time to assess the correlates of early loss of individual teeth. In starting from an intact dentition, prior tooth loss obviously cannot be a predictor variable.

These results paint a picture of edentulism as very much a consequence of expectations and a pattern of life.
For example, edentulism's association with periodontal diseases and a history of smoking may not be directly cause-and-effect $(29,30)$, but rather a correlation with negative behaviors and attitudes that in turn lead to acceptance of extractions as a treatment. There is a parallel with the association between ascorbic acid intake and edentulism, seen only in the younger age group. If the difference between the age groups is related to nutrition per se, then it is hard to see why lower intakes would lead to tooth loss only in younger people and not older ones. We think it more likely that sufficient ascorbic acid intake is part of an interest in diet, which in turn reflects positive health behaviors and attitudes. Such individuals are unlikely to choose extractions as acceptable dental treatment outcomes. For those with few remaining teeth who are deciding whether or not to have these last few teeth removed, there is obviously a long history of choosing extractions over restorations in the past, and dental neglect is suggested by higher OHI-S and PI scores. Such people also tend to have low incomes and relatively low levels of education. Their pattern of dental visits also appears to be less frequent, and tends to be for treatment rather than for preventive care.

Little of relevance was found with general health issues. A self-perception of poor general health initially showed some association with the incidence of edentulism; however, the association proved to be indirect. There were no clear associations with diabetes and arthritis, but that was more a function of small numbers and the uncertain quality of the data on those conditions. Questions on the effect of diabetes and arthritis on tooth loss would be better addressed in studies designed specifically for that purpose.

While the biologic variables such as periodontal condition and caries status were not strong predictors in this analysis, their role could be stronger than it appeared because of the interaction among disease, attitudes, and treatment choices. The decision to extract, presumably made jointly by patient and dentist, is influenced by more than the biologic condition of the tooth concerned. Issues such as the condition of other teeth; the beliefs, desires, and expectations of the patient; the knowledge and attitudes of the dentist; and the relationship between the dentist and the patient all affect the outcome, as does the cost of alternative treatments. Tooth loss, and eventually edentulism, is not simply a biologic process; therefore, it is unreasonable to expect it to have clear-cut biological correlates.

The findings in this study suggest that the best way to keep people from losing their last few permanent teeth is to keep them from losing their first few. This may sound trite, but it emphasizes the importance of those health objectives for the year 2000 regarding tooth retention and disease prevention in childhood. To reduce edentulism in the long run, the most fruitful area of behavioral research will be in understanding the reasons for losing 
individual teeth prior to edentulism, rather than studying the loss of the last few teeth.

\section{Acknowledgment}

The authors acknowledge the contributions of Dr. Chanchai Hosanguan in carrying out some of the analyses in this report.

\section{References}

1. Hyatt TP. Report of an examination made of 2,101 high school girls. Dent Cosmos 1920:52:507-11.

2. Hyatt TP. Prophylactic odontotomy. Dent Cosmos 1923;65:234-41.

3. Dunning $\jmath M, K$ lein $H$. Saving teeth among home office employees of the Metropolitan Life Insurance Company. J Am Dent Assoc 1944:31:1632-42.

4. Knutson JW, Klein H. Studies on dental caries. IV. Tooth mortality in elementary schoolchildren. Public Health Rep 1938;53:1021-32.

5. Nizel AE, Bibby BG. Geographic variations in caries prevalence in soldiers. J Am Dent Assoc i944;31:1619-26.

6. Schlack CA, Restarski JS, Dochterman EF. Dental status of 71,015 naval personnel at first examination in 1942. J Am Dent Assoc 1946:33:1141-6.

7. US Public Health Service, National Insitiute of Dental Research. Oral health of United States adults; national findings. NIH pub no 87-2868. Washington, DC: Government Printing Office, 1987.

8. Weintraub JA, Burt BA. Tooth loss in the United States. J Dent Educ 1985;49:368-76.

9. Burt BA, Eklund SA. The dentist, dental practice, and the community. 4th ed. Philadephia: Saunders, 1992.

10. Cutress TW, Hunter PBV, Davis PB, Beck DJ, Croxson LJ. Adult oral health and attitudes to dentistry in New Zealand 1976. Wellington, NZ: Medical Research Council, 1979.

11. Gray PG, Todd JE, Slack GL, Bulman JS. Adult dental health in England and Wales, 1968. London:Her Majesty's Stationery Office, 1970.

12. Todd JE, Whitworth A. Adult dental health in Scotland, 1972. London: Her Majesty's Stationery Office, 1974.

13. US Public Health Service, National Center for Health Statistics. Edentulous persons, United States 1971. DHEW pub no (HRA) 74-1516, series 10, no 89. Washington, DC: Government Printing Office, 1974 .

14. Hunt RJ, Beck JD, Lemke JH, Kohout FJ, Wallace RB. Edentulism and oral heal th problems among elderly rural lowans: the lowa $65+$ rural health study. Am J Public Health 1985;75:1177-81.

15. US Public Health Service, National Center for Health Statistics. Plan and operation of the health and nutrition examination survey. Washington, DC: Government Printing Office, 1973. (Vital and health statistics; series 1 ; no 10a.)

16. USPublic Health Service, National Center for Health Statistics. Plan and operation of the health and nutrition examination survey. Washington, DC: Government Printing Office, 1973. (Vital and health statistics; series 1; no 10b.)

17. US Public Health Service, National Center for Health Statistics. Plan and operation of the NHANES I augmentation survey of adults 25-74 years, United States, 1974-1975. Washington, DC: Government Printing Office, 1978. (Vital and health statistics; series 1; no 14.)

18. Klein H, Palmer CE, Knutson JW. Studies on dental caries: I. Dental status and dental needs of elementary schoolchildren. Public Health Rep 1938;53:751-65.

19. Russell AL. A system of scoring for prevalence surveys of periodontal disease. J Dent Res 1956:35:350-9.

20. Greene JC, Vermillion JR. The simplified oral hygiene index. J Am Dent Assoc 1964;68:7-13.

21. USPublic Health Service, National Center for Health Statistics. Diet and dental health, a study of relationships. Washington, DC: Government Printing Office, 1982; DHHS pub no (PHS) 82-1675, series 11 ; no 225

22. US Public Health Service, National Center for Health Statistics. Plan and operation of the NHANES I epidemiologic follow-up study: 1982-1984. Washington, DC: Government Printing Office, 1987. (Vital and health statistics; series 1; no 22.)

23. Cornoni-Huntley J, Barbano HE, Brody JA, et al. National Health and Nutrition Examination I - epidemiologic followup survey. Public Health Rep 1983;98:245-51.

24. Madans JH, Kleinman JC, Cox CS, et al. Ten years after NHANES I: report of initial followup, 1982-84. Public Health Rep 1986;101: $465-73$.

25. US Public Health Service, National Center for Health Statistics. A statistical methodology for analyzing data from a complex survey: the first national health and nutrition examination survey. Washington, DC: Government Printing Office, 1982. (Vital health and statistics; series 2 ; no 92 .)

26. Institute for Social Research, Survey Research Center Computing Section. OSIRIS IV user's manual. Ann Arbor: Institute for Social Research, 1981.

27. Iowa State University, Statistical Laboratory. PC CARP, Version (computer program). Ames: Iowa State University, 1989.

28. SAS Institute. PROC LOGISTIC (computer program). Cary, NC: SAS Institute, 1988.

29. Ismail AI, Burt BA, Eklund SA. Epidemiologic patterns of smoking and periodontal disease in the United States. I Am Dent Assoc 1983;106:617-21.

30. Burt BA, Ismail AI, Morrison EC, Beltran ED. Risk factors for tooth loss over a 28-year period. J Dent Res 1990;69:1126-30. 